\title{
miR-203 promotes proliferation, migration and invasion by degrading SIK1 in pancreatic cancer
}

\author{
ZHI-GUO REN $^{1^{*}}$, SHU-XIAO DONG ${ }^{2 *}$, PING HAN $^{2}$ and JIAN QI ${ }^{2}$ \\ ${ }^{1}$ Department of General Surgery, Affiliated Hospital of Shandong Medical College, Linyi, Shandong 276004; \\ ${ }^{2}$ The Second Department of General Surgery, Linyi People's Hospital \\ Affiliated to Shandong University, Linyi, Shandong 276003, P.R. China
}

Received May 31, 2015; Accepted July 9, 2015

DOI: $10.3892 / o r .2015 .4534$

\begin{abstract}
Pancreatic ductal adenocarcinoma (PDA) is among the most lethal human cancers and it is insensitive to many chemotherapeutic drugs. The molecular basis of pancreatic cancer remains to be elucidated. Investigations into the molecular mechanism involved in the development and progression as well as drug resistance of the disease may be useful to understand the pathogenesis and progression of the disease and offer new targets for effective therapies. In the present study, we showed that salt-inducible kinase 1 (SIK1) was downregulated and loss of SIK1 was associated with gemcitabine resistance in pancreatic cancer. In pancreatic cancer cells, SIK1 inhibited proliferation, migration and invasion. An analysis of potential microRNA target sites was performed using the prediction algorithms, miRanda, TargetScan and PicTar. The three algorithms predicted that miR-203 is capable of targeting 3'UTR of SIK1. Subsequent experiments confirmed the prediction. In addition, the results showed that miR-203 promotes proliferation, migration and invasion in pancreatic cancer cells, whereas the restoration of SIK1 abrogated the regulation of pre-miR-203-mediated proliferation, migration and invasion.
\end{abstract}

\section{Introduction}

Pancreatic cancer is a major cause of cancer mortality worldwide, with a 5 -year survival rate of $<5 \%$ (1). The outlook for patients who undergo surgical resection is improved, and in specialized centers, resection rates of $>15 \%$ can be achieved (2). However, surgery cannot guarantee a cure, and the 5-year survival rate does not exceed $10 \%$ following resection (2).

Correspondence to: Dr Shu-Xiao Dong, The Second Department of General Surgery, Linyi People's Hospital Affiliated to Shandong University, 27 Jiefang Road, Lanshan, Linyi, Shandong 276003, P.R. China

E-mail: shuxiaodong@yahoo.com

*Contributed equally

Key words: SIK1, miR-203, gemcitabine resistance, pancreatic cancer
Thus, improvement of long-term survival in these patients is crucial. Elucidating the molecular mechanism of proliferation, migration and invasion in pancreatic cancer may be useful to understand the pathogenesis and progression of the disease, and to offer new targets for effective therapies.

Salt-inducible kinase 1 (SIK1), also known as MSK/SIK/ SNF1LK is a member of the AMP-activated protein kinaserelated kinases (AMPK-RKs) (3). Cheng et al previously reported that SIK1 is a regulator of p53-dependent anoikis through the use of a kinome-wide loss-of-function screen. Additionally, SIK1 loss facilitated the metastatic spread and survival of disseminated cells as micrometastases in lungs and a decreased SIK1 expression closely correlated with the development of distal metastases in breast cancers from three independent cohorts (4). However, the roles of SIK1 have not been reported in pancreatic cancer.

The identification of microRNAs (miRNAs) has broadened our understanding of the mechanisms that regulate the gene expression with the addition of an entirely novel level of regulatory control. miRNAs are regulatory, non-coding RNAs 18-25 nucleotides in length and are expressed at specific stages of tissue development or cell differentiation, and have large-scale effects on the expression of a variety of genes at the post-transcriptional level. Through base pairing with its targeted mRNAs, a miRNA induces RNA degradation or translational suppression of the targeted transcripts (5-10). The aberrant expression of miRNAs has been reported in various human cancer types and is characterized to have an oncogenic or tumor-suppressor role. miRNAs are shown to play key roles in cell survival, proliferation, apoptosis, migration, invasion and various other characteristic features that are altered in human cancers $(11,12)$. It was previously reported that miR-203 is upregulated in pancreatic cancer tissues and the elevated expression is associated with poorer survival $(13,14)$. However, the roles mechanisms of miR-203 as an oncogene are crucial in pancreatic cancer.

In the present study, we showed that SIK1 is downregulated and loss of SIK1 was associated with gemcitabine resistance in pancreatic cancer. In pancreatic cancer cells, SIK1 inhibited proliferation, migration and invasion. We performed an analysis of potential microRNA target sites using the prediction algorithms, miRanda, TargetScan and PicTar. The three algorithms predicted that miR-203 is capable of targeting 3'UTR 
of SIK1, as confirmed by subsequent experiments. In addition, miR-203 was found to promote proliferation, migration and invasion in pancreatic cancer cells, whereas the restoration of SIK1 abrogated the regulation of pre-miR-203-mediated proliferation, migration and invasion.

\section{Materials and methods}

Pancreatic tissues. Pancreatic cancer tissues and adjacent normal tissues were obtained from the Second Department of General Surgery, Linyi People's Hospital Affiliated to Shandong University. The tissues were examined histologically, and pathologists confirmed the diagnosis. The medical Ethics Committee of Linyi People's Hospital Affiliated to Shandong University approved the experiments undertaken. The use of human tissue samples was conducted as per internationally recognized guidelines and local and national regulations. Informed consent was obtained from each individual.

Cell culture. L3.6pl, BxPC-3, CFPAC, MiaPaCa-2, ASPC-1, PANC-1, MPanc96, HPAC, SU86.86 and HS766T pancreatic cancer cell lines were obtained from the American Type Culture Collection (ATCC; Manassas, VA, USA). Briefly, the cells were maintained in RPMI-1640 medium supplemented with $10 \%$ fetal bovine serum (FBS; Gibco, Grand Island, NY, USA) and penicillin/streptomycin at $37^{\circ} \mathrm{C}$ in a humidified atmosphere with $5 \% \mathrm{CO}_{2}$.

Western blot analysis. Western blot analysis was performed as previously described (15). Briefly, the cells were incubated with the primary antibodies, including anti-SIK1 (1:250), antic-myc (1:250), anti-Ki67 (1:250), anti-PCNA (1:250), anti-p21 (1:250), anti-p53 (1:250), anti-RB (1:250), anti-CDK1 (1:250), anti-CDK2 (1:250), anti-CDK4 (1:250), anti-CDK6 (1:250); anti-CD44 (1:250), anti-Tspan8 (1:250) and anti- $\beta$-actin (1:500) (all from Abcam, Cambridge, MA, USA) overnight at $4^{\circ} \mathrm{C}$. IRDye ${ }^{\mathrm{TM}}-800$ conjugated anti-rabbit secondary antibodies (Li-COR Biosciences, Lincoln, NE, USA) were used for $30 \mathrm{~min}$ at room temperature. The specific proteins were visualized by the Odyssey ${ }^{\mathrm{TM}}$ Infrared Imaging system (Gene Company, Lincoln, NE, USA).

MTT assay. The effect of the cell proliferation was assessed by the 3-(4,5-dimethylthiazol-2-yl)-2,5-diphenyltetrazolium (MTT; Sigma, St. Louis, MO, USA) assay, which was performed as previously described (16). Absorbance was directly proportional to the number of survival cells.

Cell cycle analysis. Cell cycle analysis was performed as

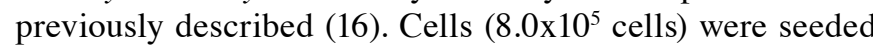
in a $100-\mathrm{mm}$ culture plate and allowed to attach overnight. The cells were transfected with plasmids for $24 \mathrm{~h}$, washed twice with $\mathrm{NaCl} / \mathrm{Pi}$, and then centrifuged at $200 \mathrm{x}$ g at room temperature. The pellet was resuspended in $1 \mathrm{ml}$ cold $\mathrm{NaCl} / \mathrm{Pi}$ and fixed in $70 \%$ ethanol for at least $12 \mathrm{~h}$ at $4^{\circ} \mathrm{C}$. The fixed cells were incubated with $100 \mu \mathrm{l}$ DNase-free RNase A $(200 \mu \mathrm{g} / \mathrm{ml})$ for $30 \mathrm{~min}$ at $37^{\circ} \mathrm{C}$, and then $1 \mathrm{mg} / \mathrm{ml}$ propidium iodide was added. The stained cells were analyzed using a fluorescenceactivated cell sorter (BD Accuri C6; BD Biosciences, Ann Arbor, MI, USA). The percentages of cells in the G1, S and
G2/M phases of the cell cycle were determined using CellQuest Pro software (FlowJo, Ashland, OR, USA).

Bromodeoxyuridine labeling and immunofluorescence. Bromodeoxyuridine labeling and immunofluorescence was performed as previously described (16). The cells grown on coverslips (Fisher, Pittsburgh, PA, USA) were incubated with bromodeoxyuridine (BrdU) for $1 \mathrm{~h}$ and stained with anti-BrdU antibody (Upstate, Temecula, CA, USA) according to the manufacturer's instructions. Images were obtained under a laser scanning microscope (Axioskop 2 Plus; Carl Zeiss Co., Ltd., Jena, Germany).

Colony formation. Colony formation was performed as previously described (16). For the colony formation assay, the cells were transfected as indicated, and then seeded in a 6-well plate. FBS $(0.2 \mathrm{ml})$ was added per well on day 5. After 9-10 days incubation, plates were washed with PBS and stained with $0.1 \%$ crystal violet. Colonies with over 50 cells were manually counted.

Reverse transcription-polymerase chain reaction and quantitative polymerase chain reaction for $m R N A$. Total RNA was isolated from the cells or tissues using TRIzol reagent (cat no. 101472; Invitrogen Life Technologies, Carlsbad, CA, USA). cDNA was synthesized from $1 \mu \mathrm{g}$ of total RNA in a $20-\mu 1$ reverse transcription (RT) system followed by PCR amplification in a 50- $\mu 1$ PCR system performed using an RT-PCR kit (cat no. A3500; Promega, Madison, WI, USA). Housekeeping gene glyceraldehyde-3-phosphate dehydrogenase $(G A P D H)$ was used as RNA loading control. The PCR primer sequences used were: GAPDH forward, 5'-ATTCAACGGCACAGTC AAGG-3' and reverse, 5'-GCAGAAGGG GCGGAGATGA-3'; PCNA forward, 5'-CTGTAGCGGCGTTGTTGC-3' and reverse, 5'-TCGTTGATGAGGTCCTTG-3'; Ki67 forward, 5'-CAACTATCCTCGTCTGTCC-3' and reverse, 5'-GGTCCC TAAAGATGTGCT-3'; p21 forward, 5'-CCCGTGAGCGAT GGAACT-3' and reverse, 5'-CGAGGCACAAGGGTACA AGA-3'; p53 forward, 5'-CCTCCTCAGCATCTTATCCG-3' and reverse, 5'-CACAAACACGCACCTCAAA-3'; Rb forward, 5'-AAGGTTTCAGGGTATCAG-3' and reverse, 5'-GTGGGTCTGTATGTTGTG-3'; Tspan8 forward, 5'-TCGAATTCTTTCCGAAATGGCAGGTGTGAG-3' and reverse, 5'-ATGTCGACTGCATCCACAGATTCATTTG TTC-3'; CD44 forward, 5'-AGACATCTACCCCAGCAAC-3' and reverse, 5'-CGTTGAGTCCACTTGGCTTTC-3'; CDK2 forward, 5'-AGAAACAAGTTGACGGGAG-3' and reverse, 5'-GAAGAGGAATGCCAGTGAG-3'; CDK4 forward, 5'-CAGTTCGTGAGGTGGCTTTA-3' and reverse, 5'-GGGG TGCCTTGTCCAGATA-3'; CDK6 forward, 5'-TGCCCACTG AAACCATAAAGG-3' and reverse, 5'-ATCCACAGCGTGA CGACCA-3'; and SIK1 forward, 5'-GTCCCTCGGAAGGAA CTAGC-3' and reverse, 5'-CTCGCGTTTTTCCTTAGCTG-3'. PCR was conducted according to the manufacturer's instructions and the PCR products were analyzed by agarose gel electrophoresis. The gels were photographed and densities of the bands were determined with a computerized image analysis system (Alpha Innotech, San Leandro, CA, USA). The area of each band was calculated as the integrated density value (IDV). Quantitaive PCR (qPCR) for SIK1 was performed with a Power 
A

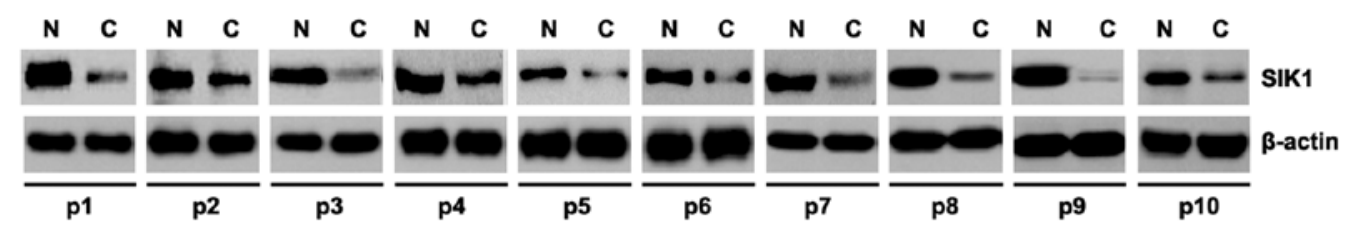

B
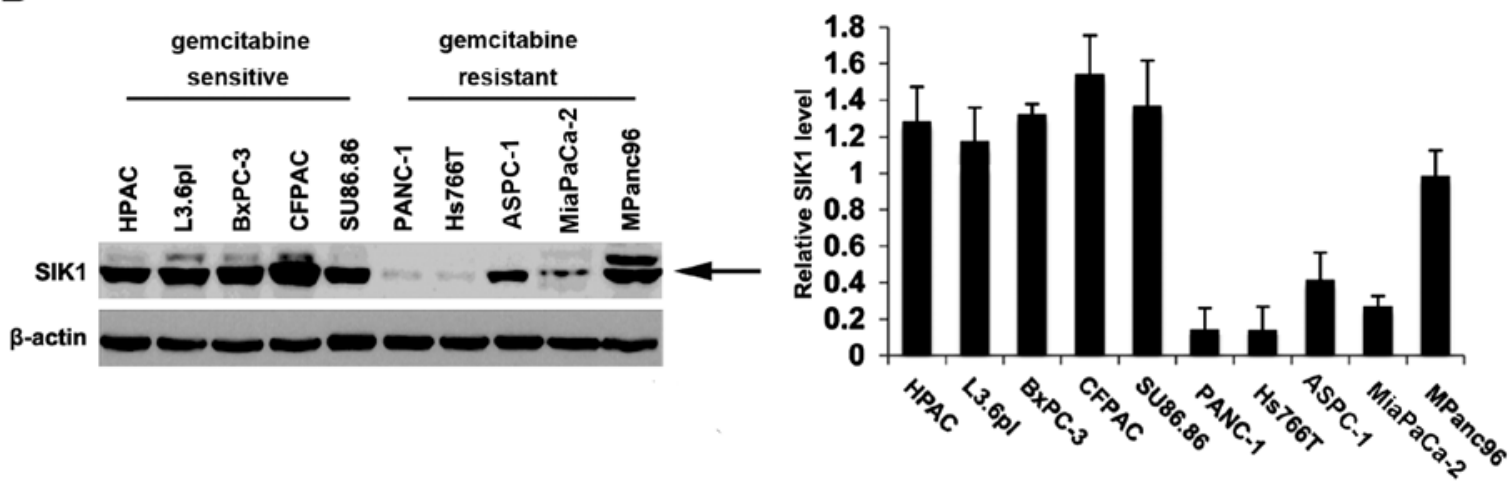

Figure 1. SIK1 is downregulated and loss of SIK1 is associtated with gemcitabine resistance in pancreatic cancer. (A) Western blot analysis for SIK1 protein in pancreatic cancer tissues and adjacent normal tissues. $\beta$-actin was used as the loading control; $n=10$. (B) Western blot analysis for SIK1 protein in pancreatic cancer cell lines. $\beta$-actin was used as the loading control; $n=3$. SIK1, salt-inducible kinase 1 .

SYBR-Green PCR Master Mix (Applied Biosystems, Carlsbad, CA, USA) according to the manufacturer's instructions.

qPCR for microRNAs. Total RNA from cultured cells, with efficient recovery of small RNAs, was isolated using the mirVana miRNA Isolation kit (cat no. AM2654; Ambion, Austin, TX, USA). Detection of the mature form of miRNAs was performed using the mirVana RT-qPCR miRNA Detection kit (cat no. AM7659; Ambion), according to the manufacturer's instructions. The U6 small nuclear RNA was used as an internal control.

Methods of bioinformatics. Analysis of the potential microRNA target sites was performed using the prediction algorithms, miRanda (http://www.microrna.org/), TargetScan (http://www. targetscan.org) and PicTar (http://pictar.bio.nyu.edu).

Immunofluorescence analysis. Immunofluorescence analysis was performed as previously described (16). For immunofluorescence analysis, the cells were plated on glass coverslips in 6-well plates and transfected as indicated. At $36 \mathrm{~h}$ after transfection, the coverslips were stained with the anti-SIK1 antibodies mentioned earlier. Alexa Fluor 488 goat anti-mouse IgG antibody or goat anti-rabbit IgG antibody was used as secondary antibody (Invitrogen Life Technologies). Coverslips were counterstained with DAPI (Invitrogen-Molecular Probes, Eugene, OR, USA) for the visualization of nuclei. Microscopic analysis was performed with a confocal laser-scanning microscope (Leica Microsystems, Bensheim, Germany). Fluorescence intensities were measured in a few viewing areas for 200-300 cells/coverslip and analyzed using ImageJ $1.37 \mathrm{v}$ software (http://rsb.info.nih.gov/ij/index.html).

Migration and invasion assay. For the Transwell migration assays, $2.5 \times 10^{4}-5.3 \times 10^{4}$ cells were plated in the top chamber with the non-coated membrane (24-well insert, pore size, $8 \mathrm{~mm}$; BD Biosciences, San Jose, CA, USA). For the invasion assays, $1.25 \times 10^{5}$ cells were plated in the top chamber with Matrigel-coated membrane (24-well insert, pore size, $8 \mathrm{~mm}$; BD Biosciences, San Jose, CA, USA). In the two assays, the cells were plated in medium without serum or growth factors, and medium supplemented with serum was used as a chemoattractant in the lower chamber. The cells were incubated for $24 \mathrm{~h}$ and cells that did not migrate or invade through the pores were removed by a cotton swab. Cells on the lower surface of the membrane were stained with the Diff-Quick Staining Set (Dade) and counted.

Wound-healing assay. Cells $\left(5 \times 10^{5}\right)$ were seeded onto each 35-mm glass bottom dish (MatTek Co., Ashland, MA, USA) and cultured at $37^{\circ} \mathrm{C}$ with $5 \% \mathrm{CO}_{2}$ for $24 \mathrm{~h}$. The confluent monolayer of the cells was wounded. Monolayers of cells were wounded with yellow pipette tips. After washing with warm PBS, the cells were incubated in fresh culture medium. The wounded areas were photographed at different time-points using a Nikon inverted microscope (Eclipse TE2000-U) equipped with a video camera (DS-U1) (both from Nikon, Japan).

Statistical analysis. Data are presented as mean \pm SEM. The Student's t-test (two-tailed) was used to compare two groups $(\mathrm{P}<0.05$ was considered significant).

\section{Results}

SIK1 is downregulated and its loss is associated with gemcitabine resistance in pancreatic cancer. To identify SIK1 expression between pancreatic cancer tissues and adjacent normal tissues, we performed western blot analysis in cancer versus normal tissues. Protein was isolated from 10 pairs of pancreatic cancer tissues and normal tissues (patient nos. 1-10). 
A

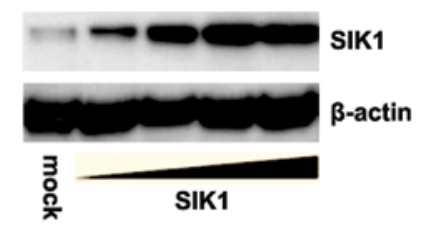

B

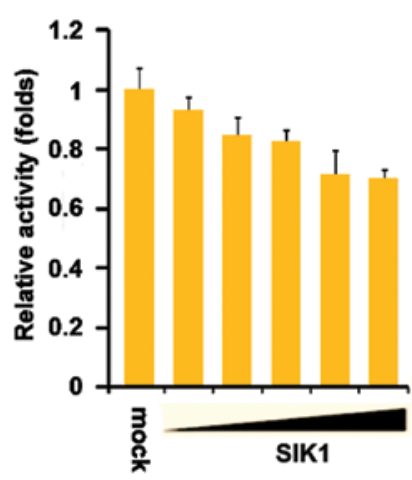

D

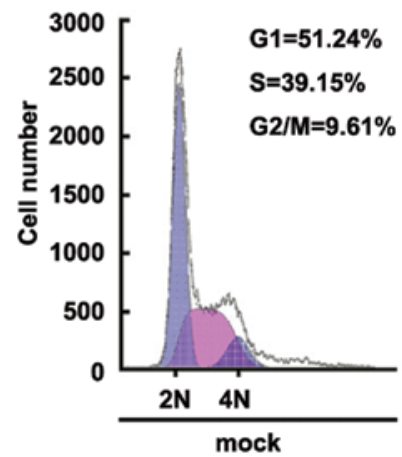

E

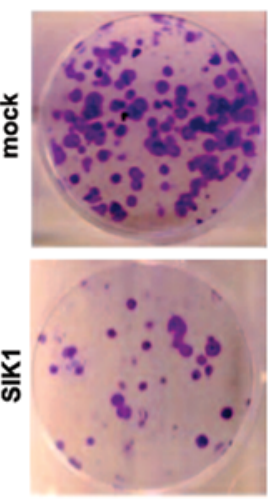

C
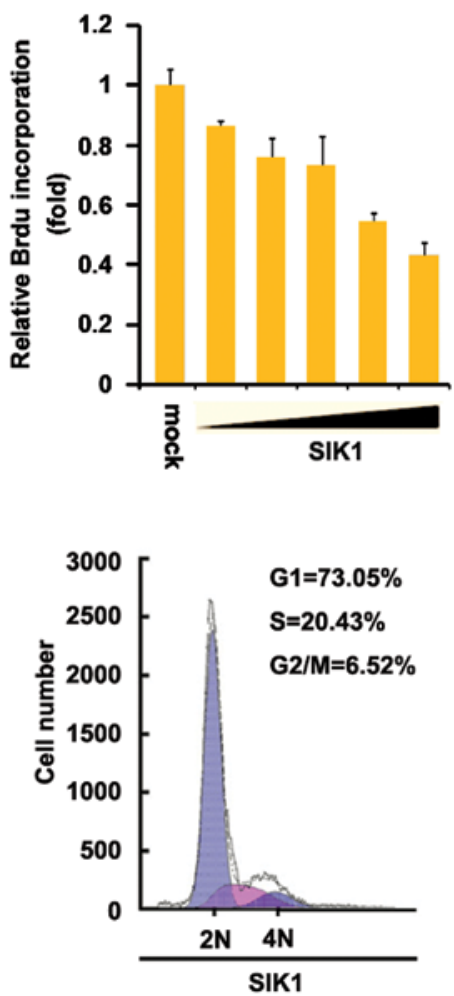

$\mathbf{F}$

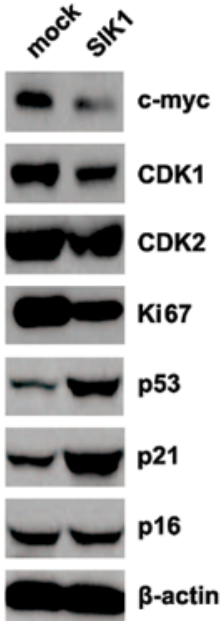

Figure 2. SIK1 inhibits proliferation in pancreatic cancer. (A) Western blot analysis for SIK1 in Hs766T cells. Hs766T cells were transfected with SIK1 expressing plasmids or control miR (mock). $\beta$-actin was used as the loading control; $\mathrm{n}=3$. (B) MTT assay for Hs766T cells. Hs766T cells were transfected with SIK1 expressing plasmids or empty vectors and then cell viability was measured at the indicated time-points by MTT assay; $\mathrm{n}=3$. (C) Brdu incorporation assay for Hs766T cells. Cells were transfected with SIK1-expressing plasmids or empty vectors (mock); n=3. (D) Cell cycle analysis for Hs766T cells transfected with SIK1 or empty vectors (mock). Histograms of DNA contents obtained by FACS analysis. The percentages of each cell cycle stage are shown in the inset of the histograms; $n=3$. (E) Colony formation assay for Hs766T cells transfected with SIK1-expressing plasmids or empty vectors (mock). Colonies with $>50$ cells were counted. Representative micrographs (left) and quantification of colonies (right) following transfection with SIK1-expressing plasmids or empty vectors (mock); n=3. (F) Western blot analysis for c-myc, CDK1, CDK2, Ki67, p21, p53 and p16 in Hs766T cells transfected with SIK1-expressing plasmids or empty vectors (mock). $\beta$-actin was used as the loading control; $n=3$. SIK1, salt-inducible kinase 1 .

We found that SIK1 protein was significantly decreased in cancer tissues, compared with adjacent normal tissues (Fig. 1A), suggesting that SIK1 is a tumor-suppressive gene in pancreatic cancer. To identify the SIK1 protein expression among different pancreatic cancer cell lines, we performed western blot analysis in the HPAC, L3.6pl, CFPAC, BxPC-3, SU86.86, 
PANC-1, Hs766T, ASPC-1, MiaPaCa-2 and MPanc96 pancreatic cancer cell lines. PANC-1, Hs766T, ASPC-1, MiaPaCa-2 and MPanc96 cells are gemcitabine-resistant cells and HPAC, L3.6pl, CFPAC, BxPC-3, SU86.86 cells are gemcitabine-sensitive cells $(17,18)$. Protein isolated from the 10 types of cell lines were detected by western blot analysis and the results showed that the expression of SIK1 was lower in gemcitabine-resistant cells (PANC-1, Hs766T, ASPC-1, MiaPaCa-2 and MPanc96 cells) than gemcitabine-sensitive cells (HPAC, L3.6pl, CFPAC, BxPC-3, SU86.86) (Fig. 1B, left panel). A quantitative image analysis was performed to analyze the SIK1 protein expression in these tissue sections and we found that SIK1 expression was indeed downregulated in gemcitabine-resistant pancreatic cancer (Fig. 1B, right panel). These results suggested that SIK1 is downregulated in pancreatic cancer tissues and associated with gemcitabine resistance.

SIK1 inhibits proliferation in pancreatic cancer. To investigate whether SIK1 affected the proliferation of pancreatic cancer cells, we examined whether SIK1-expressing plasmids stably expressed SIK1 protein in Hs766T cells, using western blot analysis. The results showed that SIK1 protein was significantly increased by SIK1-expressing plasmids in the cells (Fig. 2A). In addition, we performed an MTT assay to detect the proliferation of Hs766T cells transfected with SIK1-expressing plasmids. The results showed that SIK1 inhibited proliferation in Hs766T cells after $48 \mathrm{~h}$ of transfection and the inhibition was dose-dependent (Fig. 2B). To determine the effects of SIK1 on proliferation, we performed a Brdu incorporation assay to detect DNA synthesis in the cells. The results confirmed that SIK1 significantly inhibited DNA synthesis in the cells (Fig. 2C). To identify whether DNA synthesis inhibition contributed to lower S-phase fractions in Hs766T cells transfected with SIK1, we performed cell cycle analysis to analyze its effects on the cell cycle. The results showed lower S-phase fractions in HS766T cells transfected with SIK1 than in the cells transfected with empty vector (Fig. 2D). To identify the effect of SIK1 on colony formation, we performed the colony formation assay. The results showed that overexpression of SIK1 significantly suppressed the colony formation rate of Hs766T cells following transfection (Fig. 2E). In addition, we performed western blot analysis to confirm that SIK1 affected proliferation markers. The results of the western blot analysis demonstrated that c-myc, CDK1 and Ki67 expression were downregulated while p53 and p21 were upregulated by SIK1 (Fig. 2F).

SIK1 suppresses migration and invasion. To identify the role of SIK1 in regulating the migration and invasion of Hs766T cells, a wound-healing assay was performed. The wound-healing assay showed that SIK1 significantly inhibited motility in the cells (Fig. 3A) $(\mathrm{P}<0.05)$. To confirm the results, we performed a migration and invasion assay to detect the migration and invasion of Hs766T cells transfected with SIK1-expressing plasmids and empty vectors. Ectopic SIK1 inhibited motility and invasion by $\sim 2$-fold in the cells (Fig. 3B).

miR-203 degrades SIK1 in pancreatic cancer cells. Having demonstrated that SIK1 expression is specifically downregulated in pancreatic cancer (Fig. 1A) and it suppressed proliferation, migration and invasion in vitro, we examined the
A
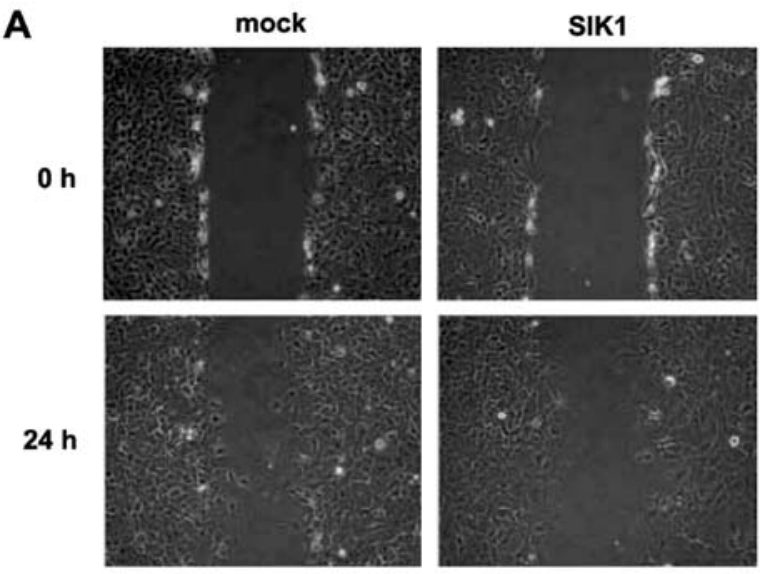

$48 \mathrm{~h}$
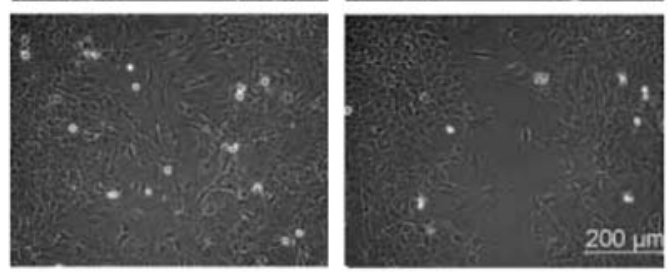

B
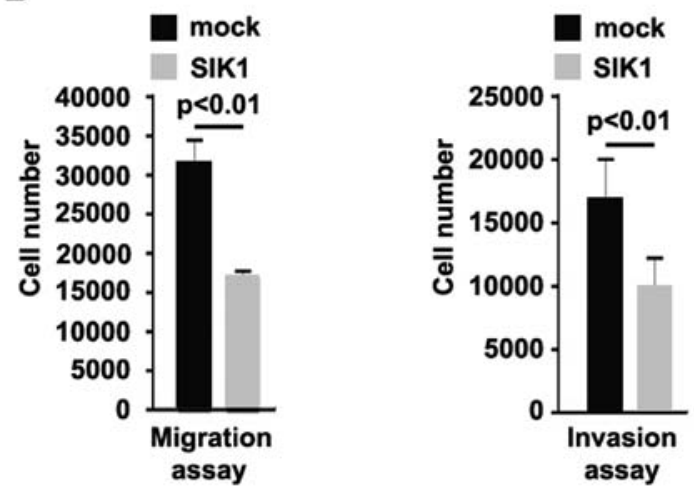

Figure 3. SIK1 inhibits migration and invasion in pancreatic cancer Hs766T cells. (A) Wound-healing assays for Hs766T cells transfected with SIK1 and empty vectors (mock); $n=3$. (B) Matrigel invasion assay and Transwell migration assay for Hs766T cells transfected with SIK1 and empty vectors (mock); $\mathrm{n}=3$. SIK1, salt-inducible kinase $1 . \mathrm{P}<0.05$.

mechanisms suppressing SIK1 expression in pancreatic cancer. MicroRNAs (miRs) are a class of small non-coding RNAs ( $\sim 22$ nucleotides) and negatively regulate the protein-coding gene expression by targeting mRNA degradation or translation inhibition (6-8). Upregulation of specific miRNA can contribute to the downregulation of tumor-suppressive gene (19-21). Thus, SIK1 was downregulated by the overexpression of specific miRNA in pancreatic cancer.

To confirm this finding, the prediction algorithms, miRanda (http://www.microrna.org/microrna/home.do), TargetScan (http://www.targetscan.org) and PicTar (http:// pictar.mdc-berlin.de/) were used to analyze 3'UTR of SIK1. The three algorithms predicted that miR-137, miR-142-3p, miR-9, miR-200a, miR-141, miR-96, miR-302a/b/c/d, miR-20, miR-203, miR-106b, miR-93, miR-106a and miR-372 were able to target 3'UTR of SIK1 (Fig. 4A). An elevated expression of miR-203 in pancreatic tumors is associated with poorer survival (13). Target sites on 3'UTR of SIK1 are shown in Fig. 4B. The result showed that miR-203 downregulated 
A TargetScan

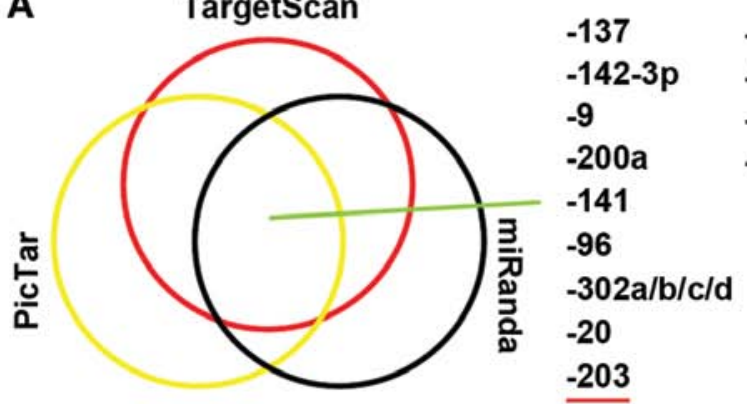

C

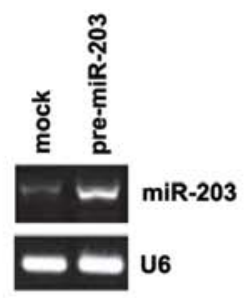

G
D

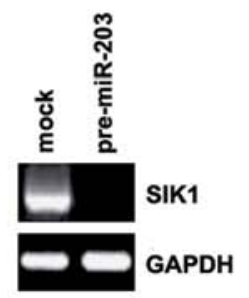

$-106 b$

$-93$

$-106 a$

$-372$

E
B

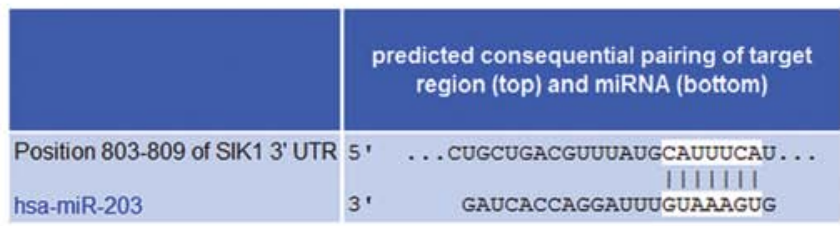

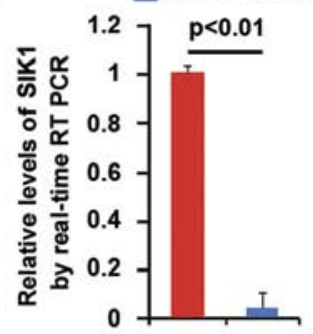

$\mathbf{F}$

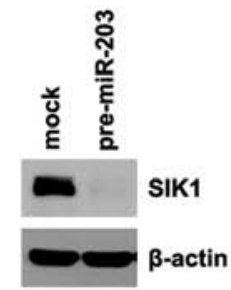

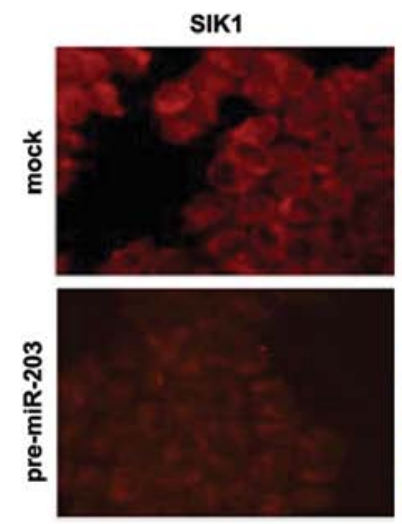
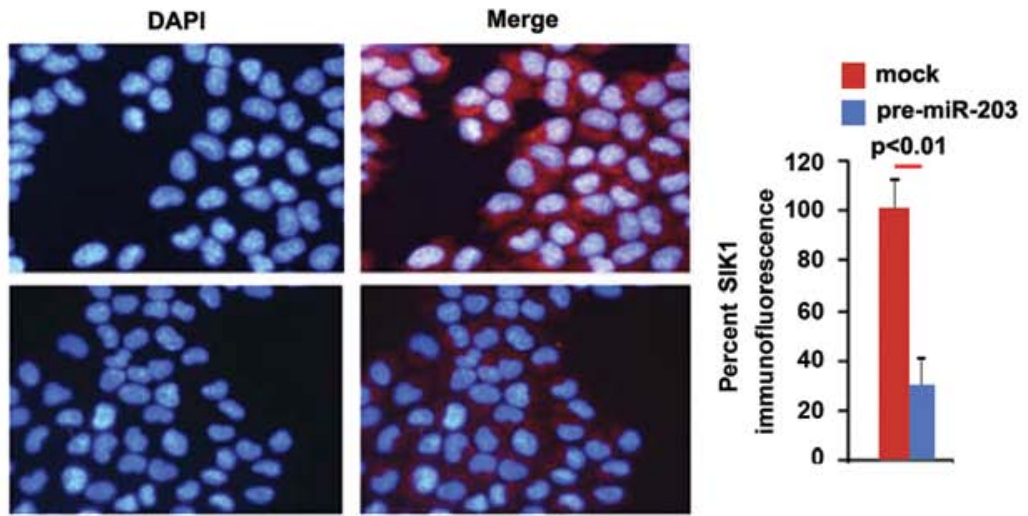

Figure 4. miR-203 degrades SIK1 in pancreatic cancer cells. (A) Vin diagram showing the predicted microRNA targeting 3'UTR of SIK1 mRNA from databases (TargetSan, miRanda and PicTar). (B) Schematic of predicted miR-203-binding sites in the 3'UTR of SIK1 mRNA by TargetScan. (C) RT-qPCR for miR-203 in BxPC-3 cells transfected with pre-miR-203 or control miR (mock). U6 was used as the loading control; n=3. (D) PCR for SIK1 in BxPC-3 cells. BxPC-3 cells were infected with pre-miR-203 or control miR (mock). GAPDH was used as the loading control; $n=3$. (E) qPCR for SIK1 in BxPC-3 cells. BxPC-3 cells were infected with pre-miR-203 or control miR (mock). GAPDH was used as the loading control; $n=3$. (F) Western blot analysis for SIK1 protein in BxPC-3 cells infected as indicated. $\beta$-actin was a loading control; $n=3$. (G) Immunofluorescence analysis for BxPC-3 cells transfected with pre-miR-203 and control miR (mock). Left panel shows microscopic pictures of immunofluorescence staining of one representative experiment (x100 magnifications). Right panel shows graphic presentation of mean fluorescence intensities; $n=3$. SIK1, salt-inducible kinase 1 .

SIK1 expression by targeting its 3'UTR in pancreatic cancer and that SIK1 was downregulated in pancreatic cancer cells, due to the overexpression of miR-203. To identify the role of miR-203 in regulating SIK1 expression in pancreatic cancer cells, BxPC-3 cells were transfected with pre-miR-203 and control miR. Following transfection, miR-203 expression was detected by qPCR and the results showed that miR-203 was significantly increased by pre-miR-203 in the cells (Fig. 4C). To confirm the result obtained, we performed RT-PCR to detect SIK1 mRNA expression in BxPC-3 cells transfected with pre-miR-203 or control miR, and identified that SIK1 mRNA was degraded (Fig. 4D). Consistent with the results of RT-PCR, the qPCR results demonstrated that SIK1 mRNA was reduced in BxPC-3 cells transfected with pre-miR-203, compared to the control miR-transfected groups (Fig. 4E). To detect whether SIK1 protein was affected by miR-203, we performed a western blot analysis in BxPC-3 cells transfected with pre-miR-203 or control miR. The results showed that SIK1 protein was evidently suppressed in the cells transfected with pre-miR-203 (Fig. 4F). We also performed immunoflurescence analysis to detect SIK1 expression in BxPC-3 cells transfected with pre-miR-203 or control miR. The results showed that SIK1 protein (Fig. 4G) was significantly downregulated in the cells transfected with pre-miR-203.

miR-203 promotes proliferation in pancreatic cancer cells. We identified that SIK1 was downregulated in pancreatic cancer tissues and inhibited proliferation, migration and invasion in pancreatic cancer cells, and that miR-203 degraded SIK1 in pancreatic cancer cells. Additionally, an elevated expression of miR-203 in pancreatic tumors was associated with poorer survival (13). Thus, contrary to SIK1, miR-203 may promote proliferation, migration and invasion in pancreatic cancer. 
A

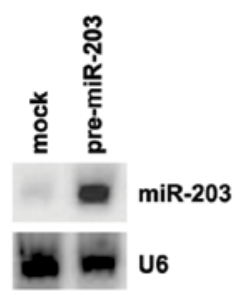

D

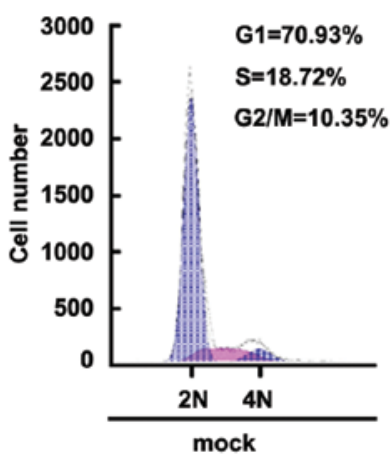

B

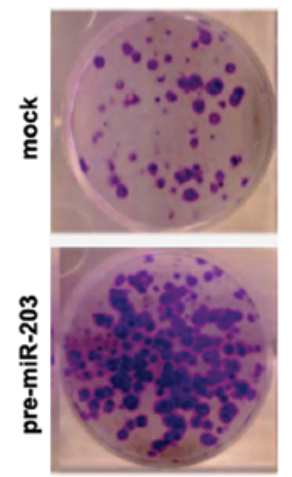

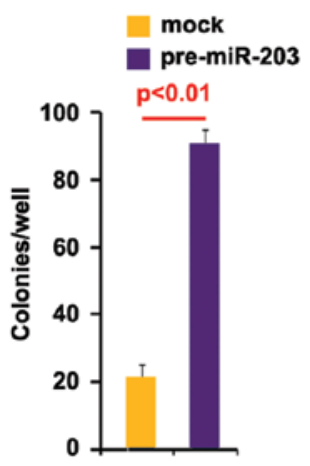

F
C

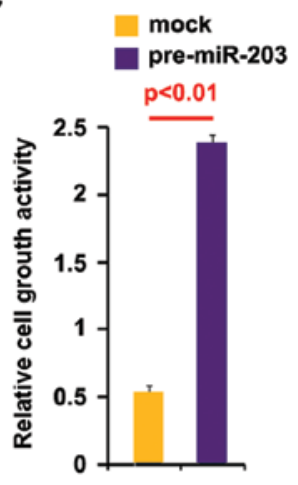

E

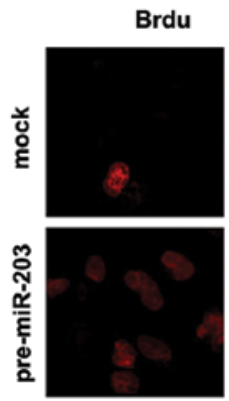

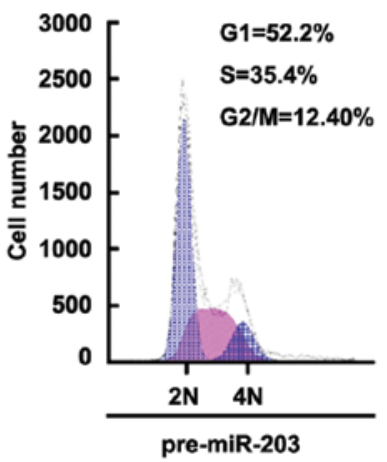

mock

pre-miR-203

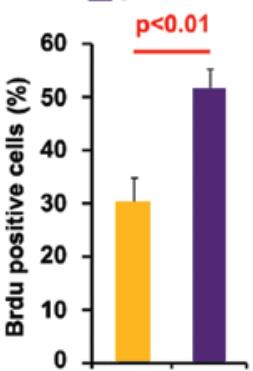

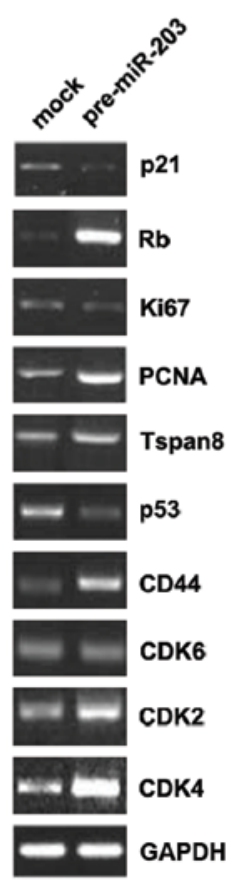

G

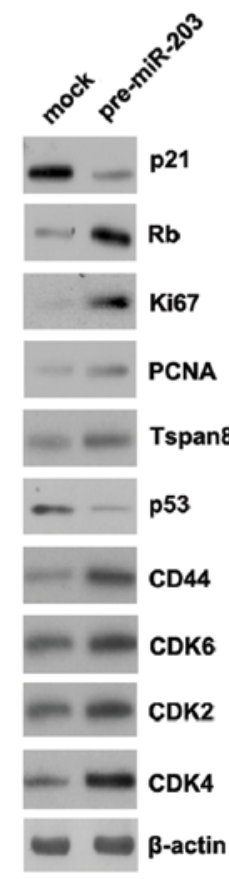

Figure 5. miR-203 promotes proliferation in pancreatic cancer cells. (A) qPCR for miR-203 in BxPC-3 cells. BxPC-3 cells were transfected with pre-miR-203 or control miR (mock). U6 was used as the loading control; n=3. (B) Colony formation assay for BxPC-3 cells transfected with pre-miR-203 or control miR (mock). Colonies with $>50$ cells were counted. Representative micrographs (left panel) and quantification of colonies (right panel) following transfection with pre-miR-203 or control miR (mock); $\mathrm{n}=3$. (C) MTT assay for BxPC-3 cells. BxPC-3 cells were transfected as indicated and then cell viability was measured by MTT assay; $n=3$. (D) Cell cycle analysis for BxPC-3 cells transfected with pre-miR-203 or control miR (mock). Histograms of DNA contents obtained by FACS analysis. The percentages of each cell cycle stages are shown in the inset of the histograms; $n=3$. (E) Brdu incorporation assay for BxPC-3 cells. Representative micrographs (left panel) and quantification (right panel) of BrdU-incorporating cells following transfection with pre-miR-203 or control miR (mock); n=3. (F) RT-PCR for p21, Rb, Ki67, PCNA, Tspan8, p53, CD44, CDK4, CDK6 and CDK2 in BxPC-3 cells infected with pre-miR-203 or control miR (mock). GAPDH was used as the loading control; $\mathrm{n}=3$. (G) Western blot analysis for p21, Rb, Ki67, PCNA, Tspan8, p53, CD44, CDK4, CDK6 and CDK2 in BxPC-3 cells infected with pre-miR-203 or control miR (mock). $\beta$-actin was used as the loading control; $n=3$.

To investigate whether miR-203 contributed to the proliferation of pancreatic cancer cells, we examined whether pre-miR-203 was stably expressed in BxPC-3 cells using qPCR. The results showed that miR-203 was significantly increased by pre-miR-203 in the cells (Fig. 5A).

To identify the effect of miR-203 on colony formation, we performed the colony formation assay. The results showed that the overexpression of miR-203 significantly increased the colony formation rate of BxPC-3 cells following transfection (Fig. 5B).

In addition, an MTT assay was performed to detect the proliferation of BxPC-3 cells transfected with pre-miR-203. The results showed that pre-miR-203 promoted proliferation in the cells after $48 \mathrm{~h}$ of transfection (Fig. 5C). To determine the effects of pre-miR-203 on proliferation, we performed a cell cycle analysis to analyze its effects on the cell cycle. The results showed higher S-phase fractions in BxPC- 3 cells transfected with pre-miR-203 than in BxPC-3 cells transfected with control miR (Fig. 5D). To identify whether DNA synthesis promotion contributed to higher S-phase fractions in BxPC-3 cells transfected with pre-miR-203, we performed the BrdU incorporation assay to detect DNA synthesis in the cells. The results confirmed that pre-miR-203 significantly promoted DNA synthesis in the cells and representative micrographs (left panel) and quantification (right panel) of BrdU-incorporating cells following transfection with pre-miR-203 or control miR 
A

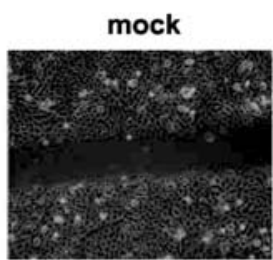

$18 \mathrm{~h}$

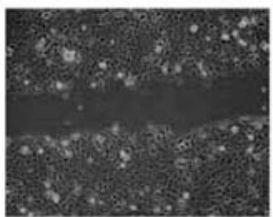

$36 \mathrm{~h}$

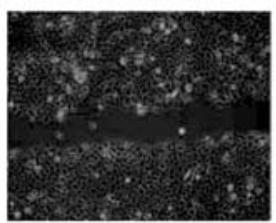

B

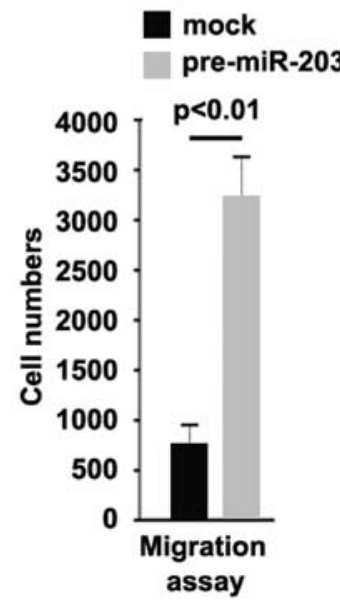

pre-miR-203
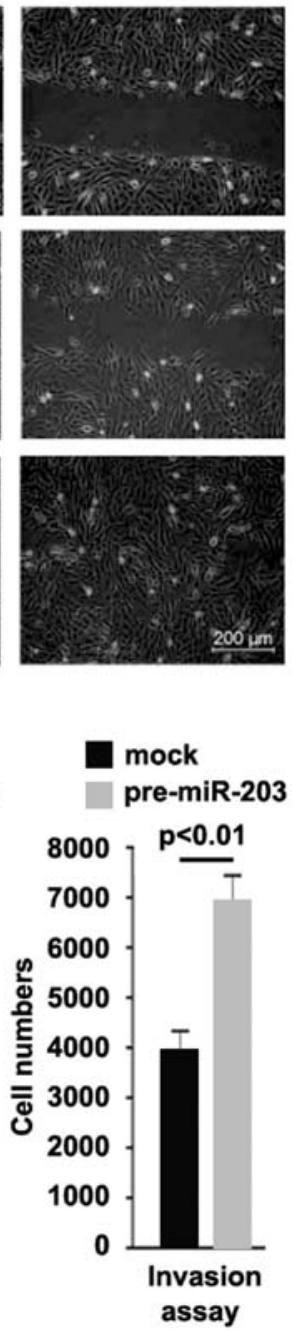

Figure 6. miR-203 promotes migration and invasion in pancreatic cancer BxPC-3 cells. (A) Wound-healing assays for BxPC-3 cells transfected with pre-miR-203 and control miR (mock); $n=3$. (B) Matrigel invasion and Transwell invasion assays for BxPC-3 cells transfected with pre-miR-203 and control miR (mock); $n=3$.

(mock) was identified (Fig. 5E). In subsequent studies, we performed RT-PCR to identify whether the mRNA of the proliferation markers was also affected by pre-miR-203 in the cells. The results of RT-PCR showed that Rb, PCNA, Tspan8, CD44, CDK2 and CDK4 expression was upregulated while p21 and p53 expression was downregulated by pre-miR-203 in the cells (Fig. 5F). In addition, we performed western blot analysis to confirm that pre-miR-203 regulated these markers. The results of the western blot analysis demonstrated that Rb, Ki67, PCNA, Tspan8, CD44, CDK2, CDK4 and CDK6 expression was upregulated while p21 and p53 expression was downregulated by pre-miR-203 (Fig. 5G). These results suggested that pre-miR-203 promotes proliferation in BxPC-3 cells.

miR-203 overexpression promotes migration and invasion in pancreatic cancer. To identify whether miR-203 affected migration and invasion, we performed a wound-healing assay, and the results showed that miR-203 significantly promoted migration in BxPC-3 cells (Fig. 6A). We also performed a migration and invasion assay to detect the role of miR-203,

A

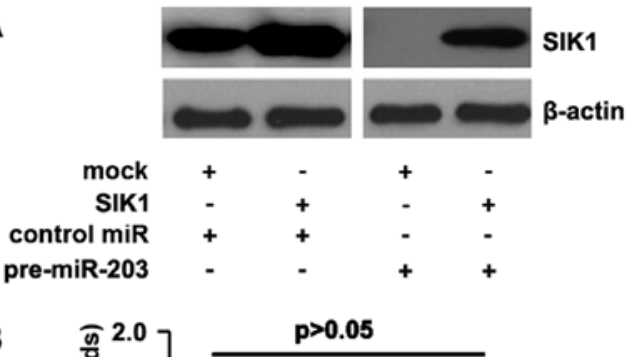

B

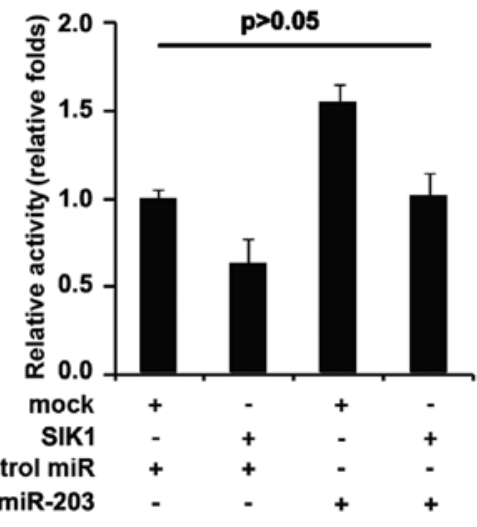

C
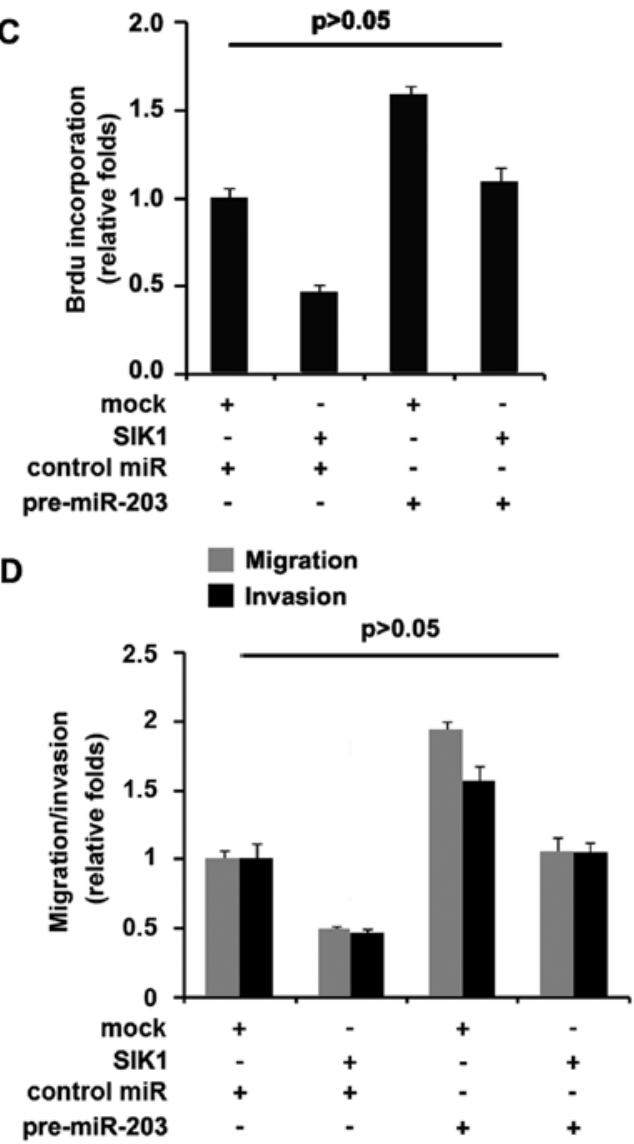

Figure 7. SIK1 abrogates pre-miR-203-mediated proliferation, migration and invasion regulation. (A) Western blot analysis for SIK1 in BxPC-3 cells transfected as indicated. $\beta$-actin was used as the loading control; $n=3$. (B) MTT assay for BxPC-3 cells. BxPC-3 cells were transfected as indicated; $n=3$. (C) BrdU incorporation assay for BxPC-3 cells. Cells were transfected as indicated; $n=3$. (D) Matrigel invasion and Transwell invasion assays for BxPC-3 cells transfected as indicated (mock); $\mathrm{n}=3$. SIK1, salt-inducible kinase 1 .

consistent with the wound-healing assay, and the results demonstrated that the ability of migration and invasion was increased in the cells transfected with pre-miR-203 (Fig. 6B). 
SIK1 abrogates pre-miR-203-mediated proliferation, migration and invasion regulation. As miR-203 degraded SIK1, we aimed to identify whether the ectopic expression of SIK1 by transfection of the cDNA that did not contain the predicted target of 3'UTR would escape the regulation of miR-203 and thus attenuate or eliminate miR-203 function. To this end, we transfected SIK1-expressing plasmids or empty vector (mock) into control miR or pre-miR-203-treated BxPC-3 cells. Immunoblotting revealed that the transfection of SIK1-expressing plasmids eliminated the effect of miR-203 on SIK1 protein (Fig. 7A). The overexpression of miR-203 in BxPC-3 cells promoted proliferation, motility and invasion. To identify whether SIK1 abrogated or attenuated the roles of miR-203 on proliferation, motility and invasion, control miR or pre-miR-203-treated BxPC-3 cells were transfected with SIK1-expressing plasmids or empty vectors (mock), we performed MTT, Brdu incorporation, and migration and invasion assays and then determined whether pre-miR-203-treated BxPC-3 cells exhibited a $>50 \%$ increase in proliferation, migration and invasion as compared to control miR-treated cells (Fig. 7B-D). Restoration of SIK1 sufficed to reverse the increase of migration and invasion (Fig. 7B-D) observed in pre-miR-203-treated cells. Thus, miR-203 promoted proliferation, migration and invasion by degrading SIK1 expression.

\section{Discussion}

The AMP-activated protein kinases (AMPKs) are important in regulating metabolism and cell growth (22-24). SIK1 is a member of AMPK-RKs (3). Clinical studies have shown that reduced levels of SIK1 are associated with distal metastases and poor outcome in breast cancer, and SIK1 expression has been associated with a tumor-suppressor function (4,25-27). In the present study we have shown that SIK1 was downregulated and loss of SIK1 was associated with gemcitabine resistance in pancreatic cancer. Thus, it is probable that SIK1 is a tumor-suppressive gene that plays an important role in gemcitabine resistance.

We identified that SIK1 was able to suppress proliferation in pancreatic cancer cells. We found that the clonogenic ability was significantly suppressed by SIK1. There is a connection between cancer stem cells and cologenic ability (28-31). The results showed that SIK1 inhibited sphere growth in pancreatic cancer (data not published). Moreover, consistent with a previous report that SIK1 couples LKB1 to p53-dependent anoikis and suppresses metastasis in breast cancer (4), we demonstrated that SIK1 overexpression significantly upregulated the p53 level and ectopically expressed SIK1-inhibited migration and invasion in pancreatic cancer cells.

Upregulation of specific miRNAs can contribute to the downregulation of tumor-suppressive genes (19-21). Thus, we examined whether SIK1 was downregulated by the overexpression of specific miRNAs in pancreatic cancer tissues and gemcitabine-resistant cell lines. miR-203 is upregulated in pancreatic cancer tissues and the elevated expression is associated with poor survival $(13,14)$. Three prediction algorithms were utilized to analyze 3'UTR of SIK1, and the results showed that miR-203 was able to target 3'UTR of SIK1, as confirmed by subsequent studies. However, further studies determining whether miR-203 and the tumor-suppressor gene
SIK1 are inversely expressed and whether miR-203 suppression mediates the upregulation of SIK1 protein in pancreatic cancer are to be conducted.

In line with previous report that miR-203 is upregulated in pancreatic cancer tissues and the elevated expression is associated with poorer survival $(13,14)$, we found that miR-203 promotes proliferation, invasion and migration in pancreatic cancer cells.

CD44 is a marker of cancer stem cells in pancreatic cancer $(32,33)$. In thre present study, the results showed that cologenic ability was significantly increased, and that CD44 was significantly upregulated by miR-203. The results suggest that miR-203 was associated with cancer stem cells. However, the roles of miR-203 in cancer stem cells of pancreatic cancer remain to be determined.

To conclude, in this study we characterized a function of SIK1 in pancreatic cancer. The results have shown that SIK1 is regulated by miR-203 and that miR-203 functions via SIK1 in pancreatic cancer.

\section{References}

1. Jemal A, Siegel R, Xu J and Ward E: Cancer statistics, 2010. CA Cancer J Clin 60: 277-300, 2010.

2. Wagner M, Redaelli C, Lietz M, Seiler CA, Friess H and Büchler MW: Curative resection is the single most important factor determining outcome in patients with pancreatic adenocarcinoma. Br J Surg 91: 586-594, 2004.

3. Bright NJ, Thornton C and Carling D: The regulation and function of mammalian AMPK-related kinases. Acta Physiol (Oxf) 196: 15-26, 2009.

4. Cheng H, Liu P, Wang ZC, Zou L, Santiago S, Garbitt V, Gjoerup OV, Iglehart JD, Miron A, Richardson AL, et al: SIK1 couples LKB1 to p53-dependent anoikis and suppresses metastasis. Sci Signal 2: ra35, 2009.

5. Bartel DP: MicroRNAs: Genomics, biogenesis, mechanism, and function. Cell 116: 281-297, 2004.

6. Lee RC, Feinbaum RL and Ambros V: The C.elegans heterochronic gene lin-4 encodes small RNAs with antisense complementarity to lin-14. Cell 75: 843-854, 1993.

7. Pasquinelli AE, Reinhart BJ, Slack F, Martindale MQ, Kuroda MI, Maller B, Hayward DC, Ball EE, Degnan B, Müller P, et al: Conservation of the sequence and temporal expression of let-7 heterochronic regulatory RNA. Nature 408: 86-89, 2000.

8. Reinhart BJ, Slack FJ, Basson M, Pasquinelli AE, Bettinger JC, Rougvie AE, Horvitz HR and Ruvkun G: The 21-nucleotide let-7 RNA regulates developmental timing in Caenorhabditis elegans. Nature 403: 901-906, 2000.

9. Lewis BP, Burge CB and Bartel DP: Conserved seed pairing, often flanked by adenosines, indicates that thousands of human genes are microRNA targets. Cell 120: 15-20, 2005.

10. Farh KK, Grimson A, Jan C, Lewis BP, Johnston WK, Lim LP, Burge CB and Bartel DP: The widespread impact of mammalian microRNAs on mRNA repression and evolution. Science 310: 1817-1821, 2005.

11. Calin GA and Croce CM: MicroRNA-cancer connection: The beginning of a new tale. Cancer Res 66: 7390-7394, 2006.

12. Farazi TA, Hoell JI, Morozov P and Tuschl T: MicroRNAs in human cancer. Adv Exp Med Biol 774: 1-20, 2013.

13. Greither T, Grochola LF, Udelnow A, Lautenschläger C, Würl P and Taubert H: Elevated expression of microRNAs 155, 203, 210 and 222 in pancreatic tumors is associated with poorer survival. Int J Cancer 126: 73-80, 2010.

14. Ikenaga N, Ohuchida K, Mizumoto K, Yu J, Kayashima T, Sakai H, Fujita H, Nakata K and Tanaka M: MicroRNA-203 expression as a new prognostic marker of pancreatic adenocarcinoma. Ann Surg Oncol 17: 3120-3128, 2010.

15. Yuan ZQ, Sun M,Feldman RI, Wang G, Ma X, Jiang C, Coppola D, Nicosia SV and Cheng JQ: Frequent activation of AKT2 and induction of apoptosis by inhibition of phosphoinositide-3-OH kinase/Akt pathway in human ovarian cancer. Oncogene 19: 2324-2330, 2000. 
16. Tang L, Chen F, Pang EJ, Zhang ZQ, Jin BW and Dong WF MicroRNA-182 inhibits proliferation through targeting oncogenic ANUBL1 in gastric cancer. Oncol Rep 33: 1707-1716, 2015.

17. Li Y, VandenBoom TG II, Kong D, Wang Z, Ali S, Philip PA and Sarkar FH: Up-regulation of miR-200 and let-7 by natural agents leads to the reversal of epithelial-to-mesenchymal transition in gemcitabine-resistant pancreatic cancer cells. Cancer Res 69: 6704-6712, 2009.

18. Arumugam T, Ramachandran V, Fournier KF, Wang H, Marquis L, Abbruzzese JL, Gallick GE, Logsdon CD, McConkey DJ and Choi W: Epithelial to mesenchymal transition contributes to drug resistance in pancreatic cancer. Cancer Res 69: 5820-5828, 2009.

19. Meng F, Henson R, Wehbe-Janek H, Ghoshal K, Jacob ST and Patel T: MicroRNA-21 regulates expression of the PTEN tumor suppressor gene in human hepatocellular cancer. Gastroenterology 133: 647-658, 2007.

20. Zhu S, Wu H, Wu F, Nie D, Sheng S and Mo YY: MicroRNA-21 targets tumor suppressor genes in invasion and metastasis. Cell Res 18: 350-359, 2008.

21. Zhu S, Si ML, Wu H and Mo YY: MicroRNA-21 targets the tumor suppressor gene tropomyosin 1 (TPM1). J Biol Chem 282: 14328-14336, 2007.

22. Shackelford DB and Shaw RJ: The LKB1-AMPK pathway: Metabolism and growth control in tumour suppression. Nat Rev Cancer 9: 563-575, 2009.

23. Fu A and Screaton RA: Using kinomics to delineate signaling pathways: Control of CRTC2/TORC2 by the AMPK family. Cell Cycle 7: 3823-3828, 2008.

24. Mirouse V, Swick LL, Kazgan N, St Johnston D and Brenman JE: LKB1 and AMPK maintain epithelial cell polarity under energetic stress. J Cell Biol 177: 387-392, 2007.

25. Wang Y, Klijn JG, Zhang Y, Sieuwerts AM, Look MP, Yang F, Talantov D, Timmermans M, Meijer-van Gelder ME and Yu J: Gene-expression profiles to predict distant metastasis of lymph-node-negative primary breast cancer. Lancet 365: 671-679, 2005.
26. Chin K, DeVries S, Fridlyand J, Spellman PT, Roydasgupta R, Kuo WL, Lapuk A, Neve RM, Qian Z, Ryder T, et al: Genomic and transcriptional aberrations linked to breast cancer pathophysiologies. Cancer Cell 10: 529-541, 2006.

27. Lu X, Lu X, Wang ZC, Iglehart JD, Zhang X and Richardson AL: Predicting features of breast cancer with gene expression patterns. Breast Cancer Res Treat 108: 191-201, 2008.

28. Ross AA, Cooper BW, Lazarus HM, Mackay W, Moss TJ, Ciobanu N, Tallman MS, Kennedy MJ, Davidson NE, Sweet D et al: Detection and viability of tumor cells in peripheral blood stem cell collections from breast cancer patients using immunocytochemical and clonogenic assay techniques. Blood 82: 2605-2610, 1993.

29. Ma S, Chan KW, Hu L, Lee TK, Wo JY, Ng IO, Zheng BJ and Guan XY: Identification and characterization of tumorigenic liver cancer stem/progenitor cells. Gastroenterology 132: 2542-2556, 2007.

30. Al-Hajj M, Wicha MS, Benito-Hernandez A, Morrison SJ and Clarke MF: Prospective identification of tumorigenic breast cancer cells. Proc Natl Acad Sci USA 100: 3983-3988, 2003.

31. Liu C, Kelnar K, Liu B, Chen X, Calhoun-Davis T, Li H, Patrawala L, Yan H, Jeter C, Honorio S, et al: The microRNA miR-34a inhibits prostate cancer stem cells and metastasis by directly repressing CD44. Nat Med 17: 211-215, 2011.

32. Li C, Heidt DG, Dalerba P, Burant CF, Zhang L, Adsay V, Wicha M, Clarke MF and Simeone DM: Identification of pancreatic cancer stem cells. Cancer Res 67: 1030-1037, 2007.

33. Hermann PC, Huber SL, Herrler T, Aicher A, Ellwart JW, Guba M, Bruns CJ and Heeschen C: Distinct populations of cancer stem cells determine tumor growth and metastatic activity in human pancreatic cancer. Cell Stem Cell 1: 313-323, 2007. 